Ann. of Math. vol. 37 (1936) pp. 443-447.

2. H. Brunn, Über Kerneigebiete, Math. Ann. vol. 73 (1913) pp. 436-440.

3. Nelson Dunford, Uniformity in linear spaces, Trans. Amer. Math. Soc. vol. 44 (1938) pp. 305-354.

4. I. Liberman, On some characteristic properties of convex sets, Rec. Math. (Mat. Sbornik) N.S. vol. 13 (1943) pp. 239-262. See also Mathematical Reviews vol. 6 (1945) p. 184.

5. R. L. Moore, Foundations of point set theory, Amer. Math. Soc. Colloquium Publications, vol. 13, 1932.

6. M. H. A. Newman, Elements of the topology of plane sets of points, Cambridge University Press, 1939.

University of California at los Angeles

\title{
THE SPACE $L^{\omega}$ AND CONVEX TOPOLOGICAL RINGS
}

\section{RICHARD ARENS}

1. Introduction. The motive for investigating the class $L^{\omega}$ of functions belonging to all $L^{p}$-classes has no measure-theoretic origin: it was our desire to discover whether or not in every convex metric ring $^{1} R$ one could find a system $\{U\}$ of convex neighborhoods of 0 having the property that $f, g \in U$ implies $f g \in U$. We show here that $L^{\omega}$ has no proper convex open set $U$ containing 0 and satisfying the relation $U U \subset U$, thus supplying the desired counter-example.

The significance of neighborhood systems of the type $\{U\}$ described above is made somewhat clearer by a proof that they insure the existence and continuity of entire functions (for example, the exponential function) on the topological ring $R$.

Such neighborhood systems $\{U\}$ are always present in rings of continuous real-valued functions over any space, provided that convergence means uniform convergence on compact sets.

We also consider the relation of $L^{\infty}, L^{\omega}$, and the $L^{p}$-classes, since $L^{\omega}$ does not seem ever to have been discussed as a topological and algebraic entity.

2. Notation and elementary facts. Let us consider measurable functions defined on $[0,1]$. For $p \geqq 1$ we shall consistently employ the usual notation

Received by the editors June 26, 1946.

1 More precisely, metrizable, convex, complete topological linear algebra. For these one requires continuity in both ring operations and scalar multiplication. It will appear that $L^{\omega}$ has these properties. 


$$
\|f\|_{p}=\left(\int_{0}^{1}|f(x)|^{p} d x\right)^{1 / p}
$$

even when the right side is infinite.

Therefore $L^{p}$ consists of all functions $f$ for which $\|f\|_{p}$ is less than $\infty$. $L^{\omega}$ evidently consists of all functions $f$ for which $\|f\|_{1},\|f\|_{2}, \cdots$, $\|f\|_{p}, \cdots$ are all finite.

Because of the relation ${ }^{2}$

$$
\|f g\|_{p} \leqq\|f\|_{q} \cdot\|g\|_{r}, \quad 1 / p=1 / q+1 / r,
$$

one has

$$
\|f\|_{1} \leqq\|f\|_{2} \leqq \cdots,
$$

since the measure of $[0,1]$ is 1 . Therefore we may take the sets of functions $f$,

$$
\|f\|_{p}<e
$$

where $p \geqq 1$ and $e>0$, as neighborhoods of 0 in $L^{\omega}$. These neighborhoods are convex because

$$
\|\lambda f+\mu g\|_{p} \leqq \lambda\|f\|_{p}+\mu\|g\|_{p}<e
$$

when $\lambda, \mu \geqq 0, \lambda+\mu=1$, and $\|f\|_{p},\|g\|_{p}<e$. Therefore addition is continuous in $L^{\omega}$ and, by relation $(\mathrm{H})$, multiplication is also.

Multiplication is not generally possible in $L^{p}$.

Now the inequalities above imply that the limit

$$
\lim _{p \rightarrow \infty}\|f\|_{p}=\|f\|_{\infty}
$$

always exists. (It may be infinite.) Those $f$ 's for which $\|f\|_{\infty}$ is finite form a set usually called $L^{\infty}$, and $\|f\|_{\infty}$ is taken as a norm in $L^{\infty}$. We shall employ the known fact that $\|f\|_{\infty}^{\infty}$ is also the least number $h$ such that $|f(x)|>h$ only on a set of measure zero.

Multiplication in $L^{\infty}$ is continuous, since

$$
\|f g\|_{\infty} \leqq\|f\|_{\infty}\|g\|_{\infty},
$$

from which it follows that if $U$ is any sphere about 0 , contained in the unit sphere of $L^{\infty}$, then $U U \subset U$.

3. The relation of $L^{\infty}, L^{\omega}$, and $L^{p}$. These spaces are related by successive proper inclusion.

TheOREM 1. $L^{\infty} \subset L^{\omega} \subset L^{p}$ but $L^{\infty} \neq L^{\omega} \neq L^{p}$. The identity mappings

${ }^{2}$ Cf. E. J. McShane, Integration, Princeton, 1944, for most of the facts which we assume. A formula equivalent to $(\mathrm{H})$ appears on $\mathrm{p} .186$. 
$L^{\infty} \rightarrow L^{\omega} \rightarrow L^{p}$ are continuous, but their inverses are not. $L^{\infty}$ is dense in $L^{\omega}$, and $L^{\omega}$ is dense in each $L^{p}$.

Proof. The inclusions and the continuity of the mappings are obvious.

If we define $l(x)=|\log x|$, then $l$ does not belong to $L^{\infty}$. Since $\|l\|_{p}=(p !)^{1 / p}, l \in L^{p}$ for each $p \geqq 1$, and hence $l \in L^{\omega}$. Thus $L^{\omega} \neq L^{\infty}$.

Similarly, the function with values $x^{-1 / 2 p}$ belongs to $L^{p}$, but not to $L^{2 p}$, and hence not to $L^{\omega}$.

Now let $l_{n}(x)=n^{-1}|\log x|$ or $n$, whichever is the smaller. Then $\left\|l_{n}-0\right\|_{p}<n^{-1}\|l\|_{p}$ which tends to zero as $n \rightarrow \infty$; but $\left\|l_{n}-0\right\|_{\infty}=n$, $n \rightarrow \infty$. Thus the inverse of the mapping $L^{\infty} \rightarrow L^{\omega}$ is not continuous.

A similar process applied to the function $x^{-1 / 2 p}$ yields a sequence which converges to zero in $L^{p}$ but not in $L^{4 p}$, and thus not in $L^{\omega}$.

Finally, suppose $f \in L^{\omega}$ be given. Define

$$
f_{n}(x)= \begin{cases}-n & \text { when } f(x)<-n, \\ f(x) & \text { when }-n \leqq f(x) \leqq n, \\ n & \text { when } n<f(x) .\end{cases}
$$

Then $f_{n} \rightarrow f$ in each $L^{p}$ and hence in $L^{\omega}$. Since the $f_{n}$ are taken from $L^{\infty}$ the latter is dense in $L^{\omega}$ and in each $L^{p}$, which establishes the third sentence of the theorem.

$L^{\omega}$ can be metrized, so as to be complete, by

$$
(f, g)=\sum_{p=1}^{\infty} \frac{2^{-p}\|f-g\|_{p}}{1+\|f-g\|_{p}} .
$$

4. Multiplication in $L^{\omega}$. By relation $(\mathrm{H})$, this is continuous. The following theorem shows the divergence between its properties and those of normed rings.

THEOREM 2. $L^{\omega}$ is a convex metric commutative ring with the property that if $U$ is a convex open set in $L^{\omega}$ containing 0 , and if $U U \subset U$, then $U$ coincides with the whole space $L^{\omega}$.

PRoof. There exists a $p \geqq 1$ and an $e>0$ such that $\|f\|_{p} \leqq e$ implies $f \in U$. Therefore a function $f$ having values not greater than $h$ on a set of measure not greater than $(e / h)^{p}$, and vanishing elsewhere, must lie in $U$, together with all its powers $f^{2}, f^{3}, \cdots$.

Let $h=2$, and set $m=(e / 2)^{p}$, for brevity.

Consider any function $g$ which has the value $b$ on a set $S$ of measure $a$, and vanishes elsewhere. Suppose $k$ is any integer such that $a \leqq m k$. Select an integer $n$ such that $b k \leqq 2^{n}$. Now we can cover $S$ by $k$ nonoverlapping subsets of measure not greater than $m$ and define 
functions $f_{1}, \cdots, f_{k}$, where $f_{i}$ has the value $(b k)^{1 / n}$ on the $i$ th subset of $S$, and vanishes elsewhere. Thus $f_{1}, \cdots, f_{k} \in U$, and also $f_{1}^{n}, \cdots, f_{k}^{n} \in U$. Since $U$ is convex

$$
g=\frac{1}{k} f_{1}^{n}+\cdots+\frac{1}{k} f_{k}^{n}
$$

must belong to $U$.

Now any function $g^{\prime}$ assuming only a finite number of values is a linear combination, with positive constants whose sum is 1 , of such functions as $g$. Therefore these functions lie in $U$.

Since these functions $g^{\prime}$ are known to be dense in $L^{\infty}$ and thus in $L^{\omega}$, we have $U$ a dense, open convex set in $L$. Thus $U=L^{\omega}$.

COROLLARY. The topology assigned to $L^{\omega}$ cannot be defined by any norm.

5. Entire functions in rings. Of course Theorem 2 shows more about $L^{\omega}$ than is needed for a counter-example to the proposition mentioned in the introduction, as will appear from the following theorem, and the fact that $e^{|\log x|}=1 / x$ is not summable, while $|\log x|$, as we have seen, lies in $L^{\omega}$.

THEOREM 3. If $R$ is a complete topological ring with a complete system $\{U\}$ of convex neighborhoods of zero each satisfying $U U \subset U$, and

$$
P(z)=a_{0}+a_{1} z+a_{2} z^{2}+\cdots
$$

is a power series representing an entire function, then, for each $f \in R$,

$$
P(f)=a_{0}+a_{1} f+a_{2} f^{2}+\cdots
$$

converges, and $P$ is a continuous operation on $R$ into itself.

In particular, for the exponential function, if $U$ is convex, contains zero, and UUCU,then

$$
e^{U} \subset 1+2 U .
$$

Proof. Let us first show that $P(f)$ converges. Therefore, suppose $U$ is any neighborhood of the system $\{U\}$. Let $f \in R$.

Then for some $t>0, t f \in U$. Hence $(t f)^{2},(t f)^{3}, \cdots$ will all lie in $U$.

Further, let us find $m_{0}$ so large that for $m \geqq m_{0}$

$$
\left|a_{m} t^{-m}\right|+\left|a_{m+1} t^{-m-1}\right|+\cdots
$$

is less than 1. Then, since $U$ is convex, we can deduce that for $n>m>m_{0}$,

$$
a_{m} t^{-m}(t f)^{m}+\cdots+a_{n} t^{-n}(t f)^{n}
$$


or its equivalent

$$
a_{m} f^{m}+\cdots+a_{n} f^{n}
$$

must lie in $U$.

Since $R$ is assumed complete, $P(f)$ converges to a limit.

The continuity of $P$ can be proved as follows:

where

$$
D=P(f+h)-P(f)=\sum_{n=0}^{n} a_{n+1} g_{n+1}
$$

$$
g_{n}=(f+h)^{n+1}-f^{n+1} .
$$

Let $U$ be a neighborhood of the system $\{U\}$, and suppose $f / t \in U$ where $0<t<\infty$. Select a real number $a$,

$$
a>\left|a_{1}\right|(t+1)+\left|a_{2}\right|(t+1)^{2}+\cdots, \quad a \geqq 1,
$$

and require $h$ to be so close to zero that $a h \in U$.

There is no point in writing down the expansion of $g_{n}$ since terms cannot be collected when $R$ is not commutative. However, each term will contain $h$, and if $g_{n}$ is written as a sum of products of powers of $f / t$ and $h$, the coefficients will add up to $(t+1)^{n}-t^{n}$.

Since $f / t$ and $a h$ lie in $U$, and $U U \subset U$, we have

$$
h_{n}=(t+1)^{-n} a g_{n} \in U,
$$

where, before dividing, we have replaced $(t+1)^{n}-t^{n}$ by $(t+1)^{n}$. Now $D$ is a linear combination of $h_{1}, h_{2}, \ldots$ with coefficients whose absolute values add up to less than 1 , and since $U$ is convex we conclude $D \in U$.

Therefore $P$ is continuous at $f$.

Institute for Advanced Study 